Int. Journal of Math. Analysis, Vol. 8, 2014, no. 27, 1315 - 1320

HIKARI Ltd, www.m-hikari.com

http://dx.doi.org/10.12988/ijma.2014.45147

\title{
Coincidence Point Theorem for a New Type of Contraction on Metric Spaces
}

\author{
Rakesh Batra \\ Department of Mathematics, Hans Raj College \\ University of Delhi, Delhi-110007, India \\ Sachin Vashistha \\ Department of Mathematics, Hindu College \\ University of Delhi, Delhi-110007, India \\ Rajesh Kumar \\ Department of Mathematics, Hindu College \\ University of Delhi, Delhi-110007, India \\ Copyright (c) 2014 Rakesh Batra, Sachin Vashistha and Rajesh Kumar. This is an open \\ access article distributed under the Creative Commons Attribution License, which permits \\ unrestricted use, distribution, and reproduction in any medium, provided the original work
} is properly cited.

\begin{abstract}
In this article, we introduce a new type of contraction and prove a coincidence point theorem which generalizes some known results in this area. The artile includes examples which show the validity of our result and that these contractions form a superclass of many classes of contractions known in the litrature.
\end{abstract}

Mathematics Subject Classification: 47H10, 54E50, 54H25, 55M20

Keywords: fixed point, coincidence point, $F$-contraction, $F$-g-contraction, complete metric space 


\section{Introduction}

In 2012, Wardowski [9] introduced thre concept of the $F$-contractive mapping on a metric space and proved a fixed point theorem for such a mapping on a complete metric space. In the present paper we extend the fixed point result due to Wardowski by introducing the concept of an $F-g$-contraction. We prove a coincidence point result for an $F-g$-contraction. For more study on $F$ contractions one may refer to $[3,4,7-10]$ and on coincidence points to $[1,2,5,6]$.

\section{Preliminary Notes}

Throughout the article denoted by $\mathbb{R}$ is the set of all real numbers, by $\mathbb{R}^{+}$is the set of all positive real numbers and by $\mathbb{N}$ is the set of all natural numbers. $(X, d),(X$ for short), is a metric space with a metric $d$. Let $T: X \rightarrow X$ and $g: X \rightarrow X$ be any two mappings. $T$ and $g$ are said to have a coincidence point at $x \in X$ if $T x=g x$ and then $g x$ is called a point of coincidence. Further, a point $x \in X$ is called a fixed point of $T$ if $T x=x$.

Definition 2.1. [9] Let $F: \mathbb{R}^{+} \rightarrow \mathbb{R}$ be a mapping satisfying

(F1) $F$ is strictly increasing, i. e. for all $a, b \in \mathbb{R}$ such that $a<b, F(a)<$ $F(b)$.

(F2) For each sequence $\left\{a_{n}\right\}_{n \in \mathbb{N}}$ of positive numbers $\lim _{n \rightarrow \infty} a_{n}=0$ if and only if $\lim _{n \rightarrow \infty} F\left(a_{n}\right)=-\infty$.

(F3) There exist $k \in(0,1)$ such that $\lim _{a \rightarrow 0^{+}} a^{k} F(a)=0$

A mapping $T: X \rightarrow X$ is said to be an F-contraction if there exist $\tau>0$ such that $\tau+F(d(T x, T y)) \leq F(d(x, y))$ for all $x, y \in X$ with $T x \neq T y$.

\section{Main Results}

Definition 3.1. Let $g: X \rightarrow X$ be any mapping and $F: \mathbb{R}^{+} \rightarrow \mathbb{R}$ be a mapping as in Definition 2.1. A mapping $T: X \rightarrow X$ is said to be an $F$-g-contraction if there exists $\tau>0$ such that

$$
\tau+F(d(T x, T y)) \leq F(d(g x, g y))
$$

for all $x, y \in X$ with $g x \neq g y$ and $T x \neq T y$.

(1) with different examples of mapping $F$ gives rise to contractions of various types known in the literature. Let us have a look at some of these in the following examples. 
Example 3.2. Let $F: \mathbb{R}^{+} \rightarrow \mathbb{R}$ be given by $F(a)=$ ln a. It is clear that $F$ satisfies (F1)-(F3) ((F3) for any $k \in(0,1)$ ). For any mapping $g: X \rightarrow X$ and $\lambda \in[0,1)$, a mapping $T: X \rightarrow X$ satisfying

$$
d(T x, T y) \leq \lambda d(g x, g y)
$$

for all $x, y \in X$ is an $F$-g-contraction with $\tau=\ln (1 / \lambda)$. Conversely, any $F$ $g$-contraction $T$ satisfies (2) for all $x, y \in X$ with $g x \neq g y$ and $\lambda=\exp (-\tau)$.

Example 3.3. If $F(a)=\ln a+a, a>0$ then $F$ satisfies (F1)-(F3) and also for any mapping $g: X \rightarrow X$ and $a \lambda \in[0,1)$, a mapping $T: X \rightarrow X$ satisfying

$$
d(T x, T y) e^{d(T x, T y)-d(g x, g y)} \leq \lambda d(g x, g y)
$$

for all $x, y \in X$ is an $F$-g-contraction with $\tau=\ln (1 / \lambda)$. Conversely, any $F$ $g$-contraction $T$ satisfies (3) for all $x, y \in X$ with $g x \neq g y$ and $\lambda=\exp (-\tau)$.

Example 3.4. Consider $F(a)=-1 / \sqrt{a}, a>0$. $F$ satisfies (F1)-(F3) ((F3) for any $k \in(1 / 2,1))$. For any mapping $g: X \rightarrow X$ and a real number $\lambda>0$, a mapping $T: X \rightarrow X$ satisfying

$$
d(T x, T y) \leq \frac{1}{\left(1+\lambda \sqrt{d(g x, g y)}^{2}\right.} d(g x, g y)
$$

for all $x, y \in X$ is an F-g-contraction with $\tau=\lambda$. Conversely, any F-gcontraction $T$ satisfies (4) for all $x, y \in X$ with $g x \neq g y$ and $\lambda=\tau$.

Example 3.5. Let $F(a)=\ln \left(a^{2}+a\right), a>0$. Then $F$ satisfies (F1)(F3) and for any mapping $g: X \rightarrow X$ and a real number $\lambda>0$, a mapping $T: X \rightarrow X$ satisfying

$$
d(T x, T y)(d(T x, T y)+1) \leq \lambda d(g x, g y)(d(g x, g y)+1)
$$

for all $x, y \in X$ is an $F$-g-contraction with $\tau=\ln (1 / \lambda)$. Conversely, any $F$ $g$-contraction $T$ satisfies (5) for all $x, y \in X$ with $g x \neq g y$ and $\lambda=\exp (-\tau)$.

Example 3.6. Let $X=[0, \infty)$ with $d$ as an Euclidean metric on $X$. Consider $F: \mathbb{R}^{+} \rightarrow \mathbb{R}$ given by $F(a)=\ln$ a. Let $T: X \rightarrow X$ and let $g: X \rightarrow X$ be defined by

$$
T x=\left\{\begin{array}{l}
x^{2} / 2 \text { if } 0 \leq x \leq 1 \\
0 \text { otherwise }
\end{array} \text { and } g x=\left\{\begin{array}{l}
2 x \text { if } 0 \leq x \leq 1 \\
0 \text { otherwise }
\end{array}\right.\right.
$$

Now $\frac{d\left(T\left(1-\frac{1}{n}\right), T\left(1-\frac{1}{m}\right)\right)}{d\left(1-\frac{1}{n}, 1-\frac{1}{m}\right)}=1-\frac{1}{2 n}-\frac{1}{2 m} \rightarrow 1$ as $m, n \rightarrow \infty$. Thus $T$ is not an F-contraction. But $d(T x, T y) \leq \frac{1}{2} d(g x, g y)$ for all $x, y \in X$. So $T$ is an F-gcontraction which is not an F-contraction. Thus our contractions are more general than the contracion introduced by Wardowski in [9]. 
Remark 3.7. From (F1) and (1) it is easy to conclude that every F-gcontraction $T$ on $X$ satisfies $d(T x, T y)<d(g x, g y)$, for all $x, y \in X, g x \neq g y$.

Example 3.8. Let $F: \mathbb{R}^{+} \rightarrow \mathbb{R}, G: \mathbb{R}^{+} \rightarrow \mathbb{R}$ be any two mappings satisfying (F1)-(F3). If $F(a) \leq G(a)$ for all $a>0$ and a mapping $H=G$ - $F$ is nondecreasing then every $F$-g-contraction $T$ is $G$-g-contraction. Indeed, from Remark 3.7 we have $H(d(T x, T y)) \leq H(d(g x, g y))$ for all $x, y \in X$ with $g x \neq g y$ and $T x \neq T y$. Thus, for all $x, y \in X$ with $g x \neq g y$ and $T x \neq T y$ we obtain $\tau+G(d(T x, T y))=\tau+F(d(T x, T y))+H(d(T x, T y)) \leq$ $F(d(g x, g y))+H(d(g x, g y))=G(d(g x, g y))$.

Theorem 3.9. Let $(X, d)$ be a metric space, $g: X \rightarrow X$ be a mapping and $T: X \rightarrow X$ be an $F$-g-contraction such that $T(X) \subseteq g(X)$. If either $(X, d)$ is complete with $T$ and $g$ as continuous and commuting mappings on $X$ or $g(X)$ is complete then $g$ and $T$ have a coincidence point $x^{\star} \in X$ with the unique point of coincidence $g x^{\star}$.

Proof. First, let us observe that $T$ and $g$ have at most one point of coincidence. Indeed, if $x_{1}^{*}, x_{2}^{*} \in X T x_{1}^{*}=g x_{1}^{*} \neq g x_{2}^{*}=T x_{2}^{*}$, then we get $\tau \leq F\left(d\left(g x_{1}^{*}, g x_{2}^{*}\right)\right)-F\left(d\left(T x_{1}^{*}, T x_{2}^{*}\right)\right)=0$ which is a contradiction.

In order to show that $T$ and $g$ have a coincidence point, let $x_{0} \in X$ be arbitrary and fixed. We define a sequence $\left\{x_{n}\right\}_{n \in \mathbb{N}}$ in $X$ by $g x_{n+1}=T x_{n}, n=$ $0,1, \ldots$ Let $d_{n}=d\left(g x_{n+1}, g x_{n}\right), n=0,1, \ldots$ If there exists $k \in \mathbb{N}$ for which $g x_{k+1}=g x_{k}$, then $T x_{k}=g x_{k}$ and the proof is finished. Suppose now that $g x_{n+1} \neq g x_{n}$, for every $n \in \mathbb{N}$. Then by (1), the following holds for every $n \in \mathbb{N}$ :

$$
F\left(d_{n}\right) \leq F\left(d_{n-1}\right)-\tau \leq F\left(d_{n-2}\right)-2 \tau \leq \ldots \leq F\left(d_{0}\right)-n \tau
$$

From (6), we obtain $\lim _{n \rightarrow \infty} F\left(d_{n}\right)=-\infty$ that together with (F2) gives

$$
\lim _{n \rightarrow \infty} d_{n}=0
$$

From (F3) there exists $k \in(0,1)$ such that

$$
\lim _{n \rightarrow \infty} d_{n}^{k} F\left(d_{n}\right)=0
$$

By (6), the following holds for all $n \in \mathbb{N}$ :

$$
d_{n}^{k} F\left(d_{n}\right)-d_{n}^{k} F\left(d_{0}\right) \leq d_{n}^{k}\left(F\left(d_{0}\right)-n \tau\right)-d_{n}^{k} F\left(d_{0}\right)=-d_{n}^{k} n \tau \leq 0
$$

Letting $n \rightarrow \infty$ in (9), and using (7) and (8), we obtain

$$
\lim _{n \rightarrow \infty} n d_{n}^{k}=0
$$


Now, let us observe that from (10) there exists $n_{0} \in \mathbb{N}$ such that $n d_{n}^{k} \leq 1$ for all $n \geq n_{0}$. Consequently we have

$$
d_{n} \leq \frac{1}{n^{\frac{1}{k}}}
$$

for all $n \geq n_{0}$. In order to show that $\left\{g x_{n}\right\}_{n \in \mathbb{N}}$ is a Cauchy sequence consider $m, n \in \mathbb{N}$ such that $m>n \geq n_{0}$. From the definition of the metric and from (11) we get

$$
d\left(g x_{m}, g x_{n}\right) \leq d_{m-1}+d_{m-2}+\ldots \ldots . .+d_{n}<\sum_{i=n}^{\infty} d_{i} \leq \sum_{i=n}^{\infty} \frac{1}{i \frac{1}{k}}
$$

This together with the convergence of the series $\sum_{i=1}^{\infty} \frac{1}{i \frac{1}{k}}$ implies that the sequence $\left\{g x_{n}\right\}_{n \in \mathbb{N}}$ is a Cauchy sequence. Consider the first situation where $(X, d)$ is complete and the mappings $g$ and $T$ both are continuous and commuting. Then there exists $x^{*} \in X$ such that $\lim _{n \rightarrow \infty} g x_{n}=x^{*}$. Finally, continuity and commutativity of $T$ and $g$ yield $T x^{*}=T\left(\lim _{n \rightarrow \infty} g x_{n}\right)=\lim _{n \rightarrow \infty} T g x_{n}=$ $\lim _{n \rightarrow \infty} g T x_{n}=g\left(\lim _{n \rightarrow \infty} T x_{n}\right)=g\left(\lim _{n \rightarrow \infty} g x_{n+1}\right)=g x^{*}$. That is, $T$ and $g$ have a coincidence point at $x^{*}$. In the second situation $g(X)$ is complete. So, there exists a point $x^{* *}$ in $X$ such that the Cauchy sequence $g x_{n}=T x_{n-1}$ in $g(X)$ converges to $g x^{* *}$. Since $g x_{n} \neq g x_{n+1}$ for all $n \in \mathbb{N}$, we can find a subsequence $\left\{g x_{n_{k}}\right\}$ of the sequence $\left\{g x_{n}\right\}$ such that $g x_{n_{k}} \neq g x^{* *}$ for all $k \in \mathbb{N}$. Then by Remark $3.7 d\left(T x_{n_{k}}, T x^{* *}\right)<d\left(g x_{n_{k}}, g x^{* *}\right) \rightarrow 0$ as $k \rightarrow \infty$. This implies that $T x_{n_{k}}$ and hence $T x_{n}$ converges to $T x^{* *}$. Uniqueness of limit now implies that $T x^{* *}=g x^{* *}$. This completes the proof.

Corollary 3.10. [9] Let $(X, d)$ be a complete metric space and $T: X \rightarrow X$ be an F-contraction. Then there exists a unique fixed point of $T$ in $X$.

Proof. Take $g=I_{X}$, the identity mapping on $X$ in Theorem 3.9.

Example 3.11. Let $X=[0, \infty)$ with $d$ as an Euclidean metric on $X$. Consider $F: \mathbb{R}^{+} \rightarrow \mathbb{R}$ given by $F(a)=\ln$ a. Let $T: X \rightarrow X$ and let $g: X \rightarrow X$ be defined by

$$
T x=\left\{\begin{array}{l}
\frac{x^{2}}{2} \text { if } 0 \leq x \leq 1 \\
0 \text { otherwise }
\end{array} \text { and } g x=\left\{\begin{array}{l}
x^{2} \text { if } 0 \leq x \leq 1 \\
0 \text { otherwise }
\end{array}\right.\right.
$$

Clearly $T(x)=\left[0, \frac{1}{2}\right] \subseteq g(X)=[0,1]$ and $g(X)$ is a complete subset of $X$. Now $d(T x, T y)=\frac{1}{2} d(g x, g y)$ for all $x, y \in X$. So $T$ is an F-g-contraction. Thus by Theorem 3.9, $T$ and $g$ have a coincidence point in $X$. We observe that $T$ and $g$ have a coincidence point at 0 and at every real number greater than 1 . Also 0 is the unique point of coincidence of $T$ and $g$. 


\section{References}

[1] Rakesh Batra and Sachin Vashistha, Coupled coincidence point theorems for nonlinear contractions under $(F, g)$-invariant set in cone metric spaces, Journal of Nonlinear Sciences and Applications, 6:2 (2013), 86-96.

[2] Rakesh Batra and Sachin Vashistha, Coupled coincidence point theorems for nonlinear contractions under $c$-distance in cone metric spaces, Annals of Functional Analysis, 4:1 (2013), 138-148.

[3] Rakesh Batra and Sachin Vashistha, Fixed points of an F-contraction on metric spaces with a graph, International Journal of Computer Mathematics, (2014), doi: 10.1080/00207160.2014.887700.

[4] Rakesh Batra and Sachin Vashistha, Fixed point theorem for $F_{w^{-}}$ contractions in complete metric spaces, Journal of Nonlinear Analysis and Applications, 2013(2013), doi: 10.5899/2013/jnaa-00211.

[5] T. G. Bhaskar and V. Lakshmikantham, Fixed point theorems in partially ordered metric spaces and applications, Nonlinear Analysis, 65:7 (2006), 1379-1393.

[6] V. Lakshmikanthamand and L. Cirić, Coupled fixed point theorems for nonlinear contractions in partially ordered metric spaces, Nonlinear Analysis, 70 (2009:12), 4341-4349.

[7] M. Sgroi and C. Vetro, Multi-valued F-contractions and the solution of certain mappingal and integral equations, Filomat, 27:7 (2013), 12591268, doi: 10.2298/FIL1307259S.

[8] Satish Shukla and Stojan Radenović, Some common fixed Point theorems for $F$-contraction type mappings in 0-complete partial metric spaces, Journal of Mathematics, 2013(2013), Article ID 878730, 7 pages, doi: $10.1155 / 2013 / 878730$.

[9] D. Wardowski, Fixed points of a new type of contractive mappings in complete metric spaces, Fixed Point Theory and Applications, 2012 (2012), 94, doi: 10.1186/1687-1812-2012-94.

[10] D. Wardowski and N. Van. Dung, Fixed Points Of F-Weak Contractions On Complete Metric Spaces, Demonstratio Mathematica, 47:1 (2014), 146-155.

\section{Received: June 3, 2014}

